\title{
DETERMINANTS OF VECTOR SPECIFICITY OF O'NYONG NYONG AND CHIKUNGUNYA VIRUSES IN ANOPHELES AND AEDES MOSQUITOES
}

\author{
DANA L. VANLANDINGHAM,* KONSTANTIN TSETSARKIN, KIMBERLY A. KLINGLER, CHAO HONG, \\ KATE L. MCELROY, MICHAEL J. LEHANE, AND STEPHEN HIGGS \\ Department of Pathology, University of Texas Medical Branch, Galveston, Texas; Liverpool School of Tropical Medicine, \\ Liverpool, United Kingdom
}

\begin{abstract}
The alphaviruses o'nyong nyong virus (ONNV) and chikungunya virus (CHIKV) provide a unique system to study the viral genes involved in vector specificity. ONNV infects both anopheline and culicine mosquitoes, whereas CHIKV infects only culicine mosquitoes. In this study, chimeric viruses were constructed that contained genes from both ONNV and CHIKV. These chimeras and previously described full-length infectious clones of ONNV and CHIKV were evaluated in Anopheles gambiae and Aedes aegypti mosquitoes. Virus derived from the infectious clones of ONNV and CHIKV retained the vector specificity of the parental viruses. All six of the chimeras were found to infect Ae. aegypti mosquitoes at high rates but only the chimera containing viral genes encoding all of the structural proteins of ONNV was able to infect An. gambiae mosquitoes. These data indicate that all of the viral structural proteins are necessary for ONNV to infect An. gambiae mosquitoes.
\end{abstract}

\section{INTRODUCTION}

O'nyong nyong virus (ONNV) and chikungunya virus (CHIKV) are mosquito-borne alphaviruses in the Semliki Forest antigenic complex, family Togaviridae. ${ }^{1}$ Alphaviruses contain a linear, positive sense, single-stranded RNA genome that is $\sim 11.8 \mathrm{~kb}$ in length. ${ }^{2}$ The RNA genome consists of a capped 5' non-coding region (NCR) and a $3^{\prime}$ polyadenylated NCR. There are four nonstructural proteins (nsP1-nsP4) that are encoded at the $5^{\prime}$ end. The structural proteins are encoded at the $3^{\prime}$ end of the virus from a subgenomic 26S RNA and consist of the viral capsid (C), two envelope glycoproteins (E1 and E2), and two peptides (E3 and $6 \mathrm{~K}){ }^{2} \mathrm{~A}$ total of $89 \%$ of the 11,822-nucleotide genome of ONNV (SG650) is conserved when compared with the 11,881-nucleotide genome of CHIKV (37997). Within these conserved areas, the sequence identity is between $77 \%$ and $85 \%$.

ONNV, which is transmitted by anopheline mosquitoes such as Anopheles gambiae and An. funestus, ${ }^{3-5}$ has been involved in large scale epidemics in Africa. ${ }^{6-8}$ Although no fatalities occurred, the 1959-1962 epidemic involved more than two million cases. ${ }^{3,4}$ In 1996, ONNV was the cause of another epidemic in Uganda after an absence of 35 years. ${ }^{9}$ ONNV produces symptoms such as fever, althralgia, and rash that generally last for less than one week. ${ }^{10}$

CHIKV is transmitted by Aedes furcifer mosquitoes in Africa $^{11}$ and Culex quinquefasciatus, Ae. aegypti, and Ae. albopictus mosquitoes in Asia. ${ }^{12,13}$ This virus has caused substantial epidemics in both Africa and southeast Asia. ${ }^{14,15}$ Epidemic infection with CHIKV decreased on both continents in the 1970s and 1980s, but has recently re-emerged. A 1999 outbreak in Indonesia was the first epidemic transmission of CHIKV in this location since $1982 .{ }^{16}$ A second outbreak of infection with CHIKV occurred in Indonesia from 2001 to 2003. ${ }^{13}$ After a 39-year absence, CHIKV was found to be the cause of an urban epidemic in the Democratic Republic of the Congo. ${ }^{17}$ Infection with CHIKV is characterized by fever, nausea, althralgia, and rash.

\footnotetext{
* Address correspondence to Dana L. Vanlandingham, Department of Pathology, University of Texas Medical Branch, Keiler 2.104 L 20762, 301 University Boulevard, Galveston, TX 77555-0609. E-mail:dlvanlan@utmb.edu
}

Chimeric viruses based on ONNV and CHIKV provide useful tools to study the viral genetic determinants of vector specificity. Three strains of ONNV (SG650, Gulu, and Igbo Ora), have been shown to be infectious to varying degrees to both Ae. aegypti and An. gambiae mosquitoes and to two invertebrate cell culture lines derived from Anopheles (Mos 55) and Aedes (C6/36) mosquitoes. ${ }^{18}$ ONNV strain SG650 was found to have the highest infection rate in both species of mosquitoes compared with the two other strains. CHIKV (37997) is able to infect Ae. aegypti mosquitoes and C6/36 cells; however, An. gambiae mosquitoes and Mos 55 cells have been found to be refractory to infection with this virus. ${ }^{18}$

Six chimeric viruses based on ONNV and CHIKV were developed and characterized in Ae. aegypti and An. gambiae mosquitoes. These chimeric viruses possessed nonstructural genes from CHIKV and partial or full structural genes derived from ONNV. Complimentary chimeras were also constructed in which the nonstructural genes were obtained from ONNV and the structural genes were derived from CHIKV. The ability of these viruses to infect two species of mosquitoes was compared with virus rescued from the full-length infectious clones of ONNV and CHIKV.

\section{MATERIALS AND METHODS}

Viruses. ONNV (SG650) and CHIKV (37997) were obtained from the Centers for Disease Control and Prevention arbovirus reference center in Fort Collins, Colorado and the World Reference Center for Arboviruses at the University of Texas Medical Branch in Galveston, Texas. ONNV (SG650) was isolated from human serum in Uganda in 1996 and has been passed twice in Vero cells (GenBank accession no. AF079456). ${ }^{6}$ CHIKV (37997) was originally isolated from the mosquito Ae. furcifer in Kadougou, Senegal in 1983 (GenBank accession no. AY726732). This isolate has been passed once in AP-61 (Ae. pseudoscutellaris) cells and twice in Vero cells. Stock virus was produced after a single passage in Vero cells maintained at $37^{\circ} \mathrm{C}$ in Leibovitz L-15 medium with $10 \%$ fetal bovine serum, $100 \mathrm{U} / \mathrm{mL}$ of penicillin, and $100 \mu \mathrm{g} / \mathrm{mL}$ of streptomycin. Cell supernatants were harvested when $75 \%$ of the cells showed a cytopathic effect $(3+\mathrm{CPE})$, aliquoted, and stored at $-80^{\circ} \mathrm{C}$ for use in all experiments.

Construction of infectious clones. The full-length CHIKV (37997) infectious clone (pCHIKic) has been previously de- 
scribed. ${ }^{19}$ The full-length ONNV (SG650) infectious clone pONNic-Foy, provided by K. E. Olson and B. D. Foy (Colorado State University, Fort Collins, CO), was derived from pONN.AP3, developed by Brault and others, ${ }^{20}$ was modified by replacing the $\mathrm{T} 7$ promoter with a SP6 promoter and removing the second subgenomic promoter and restriction sites, and renamed pONNic. The six chimeric viruses, shown in Figure 1, were constructed using standard polymerase chain reaction (PCR)-based cloning procedures. The PCRamplified fragments of ONNV (SG650) or CHIKV (37997) were produced using high-fidelity $\mathrm{Pfu}$ polymerase (Stratagene, La Jolla, CA). Fragments were ligated either in tandem or singly with T4 DNA ligase (Stratagene) into pCHIKic or pONNic. All plasmids were transformed into chemically competent Escherichia coli XL10-Gold cells (Stratagene), amplified, and DNA was extracted using QIAprep Spin Miniprep Kit (Qiagen, Valencia, CA). Additional information, such as maps of the clones, is available from the authors upon request.

In vitro transcription and virus production. Infectious virus from the full-length and chimeric clones was produced by linearization with Not I, which was in vitro transcribed from the SP6 promoter using the mMESSAGE mMACHINE kit (Ambion, Austin, TX) following the manufacturer's instructions. The RNA was electroporated into baby hamster kidney-21 S cells as previously described. ${ }^{21}$ Cell culture supernatant containing virus was harvested, aliquoted, and stored at $-80^{\circ} \mathrm{C}$ when cells showed $3+\mathrm{CPE}$.

Mosquitoes. The Rexville D strain of Ae. aegypti, whiteeyed Higgs variant, and the An. gambiae G3 strain were reared at $27^{\circ} \mathrm{C}$ and a relative humidity of $80 \%$ under a 16 hour light: 8-hour dark photoperiod, as previously described. ${ }^{22-24}$ Adults were supplied with a cotton wool pad soaked in a $10 \%$ sucrose solution ad libitum.

Oral infections of mosquitoes. Four-day-old adult female Ae. aegypti or An. gambiae mosquitoes were fed a blood meal

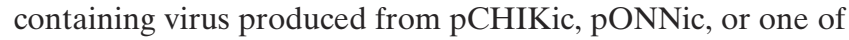
the six chimeras to be analyzed. Vero cells were inoculated with stock virus and incubated at $37^{\circ} \mathrm{C}$. Virus was harvested when $75 \%$ of the cells showed CPE. The viral supernatant was mixed with an equal volume of defibrinated sheep blood (Colorado Serum Company, Denver, CO). Adenosine triphosphate at a final concentration of $2 \mathrm{mM}$ was added to the blood meal as a phagostimulant. Mosquitoes were fed using an isolation glove box located in a Biosafety Level 3 insectary. Infectious blood was heated to $37^{\circ} \mathrm{C}$ and placed in a Hemotek feeding apparatus for one hour (Discovery Workshops, Accrington, Lancashire, United Kingdom). ${ }^{25}$ Fully engorged females were separated from unfed females and were placed into new cartons. To compare replication of the chimeric viruses, six to eight mosquitoes were removed for titration on days $1,2,3,7$, and 14 post-infection (pi) for a total of approximately 40 mosquitoes per virus examined. Mosquitoes were stored at $-80^{\circ} \mathrm{C}$ prior to titration on 96 -well plates. Day 0 blood meal and three mosquito samples, collected immediately after feeding, were used to determine the titer of virus imbibed and to evaluate continuity between experiments. At days 7 and 14 pi, salivary glands were dissected from 10 mosquitoes and stained for viral antigen to evaluate dissemination properties of the viruses.

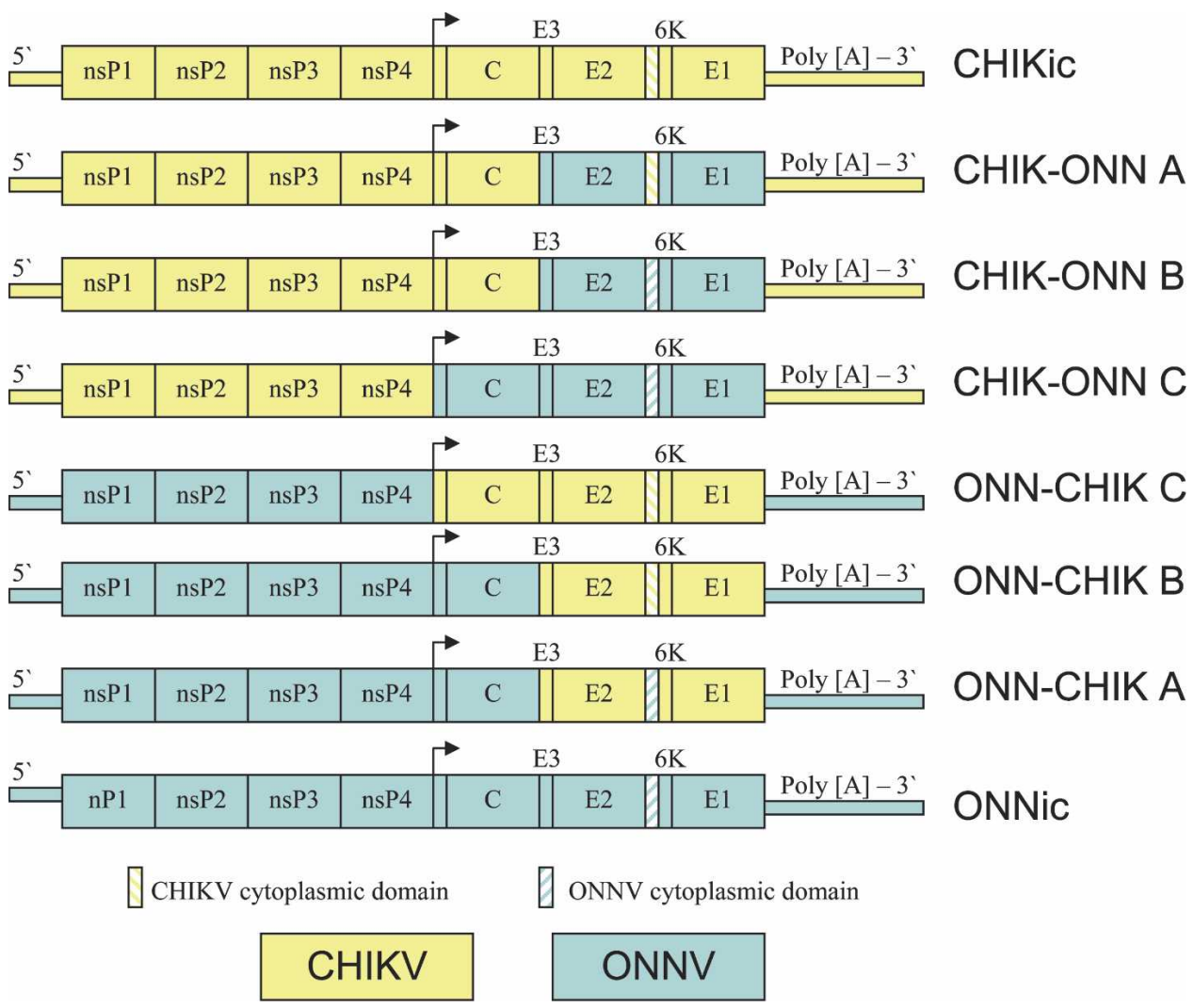

FIGURE 1. Schematic of full-length chikungunya virus infectious clone (pCHIKic), o'nyong nyong virus infectious clone (pONNic), and chimeric clones produced. Reciprocal clones have the same letter (A, B, or C) for ease of comparison. The subgenomic promoter is indicated by an arrow. nsP = nonstructural protein; $\mathrm{C}=$ capsid; $\mathrm{E}=$ envelope. This figure appears in color at www.ajtmh.org. 
Titrations. The $50 \%$ tissue culture infectious dose endpoint titers $\left(\log _{10} \mathrm{TCID}_{50} / \mathrm{mL}\right)$ were used to quantify titers of virus from cell culture and mosquito samples. ${ }^{21}$ Individual mosquitoes were triturated in $1 \mathrm{~mL}$ of L-15 medium and filtered through a $0.22-\mu \mathrm{M}$ syringe filter (Millipore, Carrigtwohill, Cork, Ireland), which was then 10-fold serially diluted in a 96-well plate seeded with Vero cells. Cells and virus were incubated at $37^{\circ} \mathrm{C}$ for seven days and then scored based on observed CPE. Differences in viral infection rates between Ae. aegypti and An. gambiae were tested for significance by Fisher's exact test using SPSS version 11.5 (SPSS Inc., Chicago, IL).

Immunofluorescence assay (IFA). Salivary glands were dissected on glass microscope slides in phosphate-buffered saline, air-dried, fixed in cold acetone for 10 minutes and stained using a cross-reactive mouse hyperimmune ascitic fluid raised against CHIKV as the primary antibody and amplifying the signal using indirect IFA protocols previously described. ${ }^{18}$

\section{RESULTS}

Virus derived from pCHIKic and pONNic were compared with CHIKV (37997) and ONNV (SG650) virus in Ae. aegypti and An. gambiae mosquitoes (Figure 2). CHIKV (37997) and virus derived from $\mathrm{pCHIKic}$ replicated at a similar rate over time, with $100 \%$ of the Ae. aegypti mosquitoes infected on most days analyzed pi (Figure 3A). Although the infection rate on day 3 pi for pCHIKic was lower than for CHIKV (37997), no significant difference $(P>0.05)$ was found between these two viruses. As expected, on day 14 pi CHIKV
(37997) and the virus derived from pCHIKic was not detected in any of the An. gambiae tested. Viral titers decrease from 6.5 or $5.8 \log _{10} \mathrm{TCID}_{50} / \mathrm{mL}$ to $0 \log _{10} \mathrm{TCID}_{50} / \mathrm{mL}$ between days 0 and 3 pi for both CHIKV (37997) and virus derived from pCHIKic (Figure 2C). The viral titer of CHIKV (37997) in An. gambiae increased to an average of $1.4 \log _{10} \mathrm{TCID}_{50} /$ $\mathrm{mL}$ with three of seven mosquitoes positive on day 7 pi. None of the An. gambiae mosquitoes examined on day 14 pi were infected (Figure 2C). Although the viral growth curves were similar, ONNV (SG650) virus infected significantly more $(P<$ $0.05)$ Ae. aegypti than virus derived from pONNic on day 14 pi (Figure 2B). However, there was no significant difference between the titers of these two viruses on day 14 pi in $A n$. gambiae (Figure 2D).

Three infectious clones containing all or part of the ONNV structural genes and CHIKV nonstructural genes and three reciprocal clones containing CHIKV structural genes and ONNV nonstructural genes were characterized in vivo in Ae. aegypti and An. gambiae mosquitoes (Figures 3 and 4). All chimeras produced were orally infectious to Ae. aegypti mosquitoes, with infection rates ranging from $56 \%$ to $100 \%$ on day 14 pi (Figure 3A and B). The infection rate in Ae. aegypti for each of the three chimeras containing the CHIKV nonstructural genes (CHIK-ONN A, B, and C) was $100 \%$ on day 14 pi (Figure $3 \mathrm{~A}$ ). The only chimera to infect An. gambiae on day 14 pi was CHIK-ONN C, which infected $38 \%$ of the $A n$. gambiae at this time point (Figure 3C). A significant difference $(P<0.05)$ in infection rates was detected between two of the chimeras containing the nonstructural genes from ONNV, ONN-CHIK A, and ONN-CHIK C in Ae. aegypti on day 14 pi (Figure 3B). There was no significant difference $(P>0.05)$ in

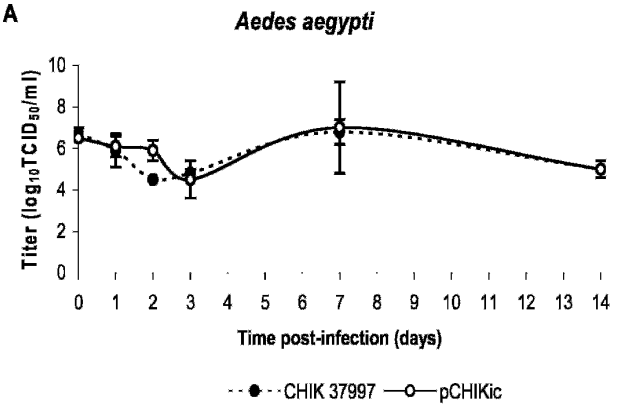

C

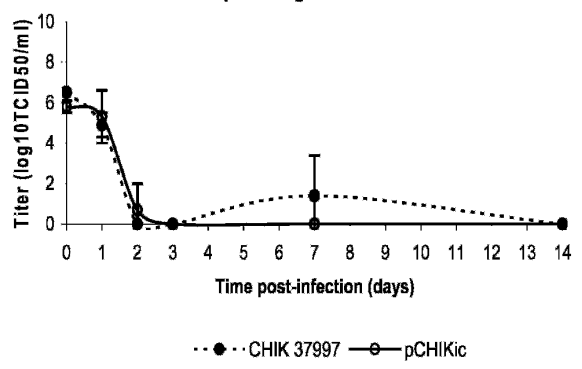

B

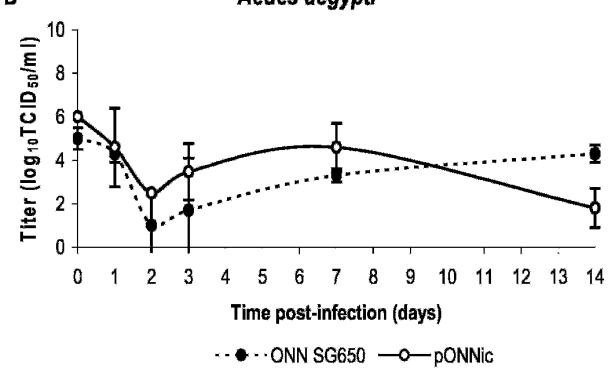

D

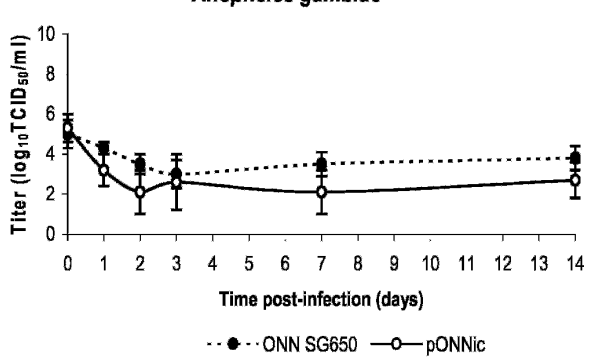

FIGURE 2. Viral titers and growth of viruses over time after a blood meal containing chikungunya virus (CHIKV) (37997), o'nyong nyong virus (ONNV) (SG650), or viruses derived from full-length pCHIKic or pONNic in Aedes aegypti (A and B) or Anopheles. gambiae (C and D) mosquitoes. Bloodmeal titers: CHIKV (37997) $7.95 \log _{10} \mathrm{TCID}_{50} / \mathrm{mL} ; *$ virus derived from pCHIKic $7.95 \log _{10} \mathrm{TCID}_{50} / \mathrm{mL}^{\dagger \dagger} \mathrm{ONNV}(\mathrm{SG} 650) 7.3$ $( \pm 0.6) \log _{10} \mathrm{TCID}_{50} / \mathrm{mL} ; *$ virus derived from pONNic $6.52 \log _{10} \mathrm{TCID}_{50} / \mathrm{mL}$. Error bars indicate variation between mosquitoes analyzed per time point: $\geq 3$ mosquitoes on day 0 post-infection (pi); $\geq 8$ mosquitoes on days 2 and 3 pi, except for CHIKV (37997); $* 8$ mosquitoes on days 1 , 7, and 14 pi, except for CHIKV (37997)*, pCHIKic. ${ }^{\dagger}$ Six or more mosquitoes were analyzed at each time point for virus derived from pONNic. $\mathrm{TCID}_{50}=50 \%$ tissue culture infectious dose. *Vanlandingham and others; ${ }^{18}{ }^{\dagger}$ Vanlandingham and others; ${ }^{19}$ Two replicates are shown for ONNV (SG650). 

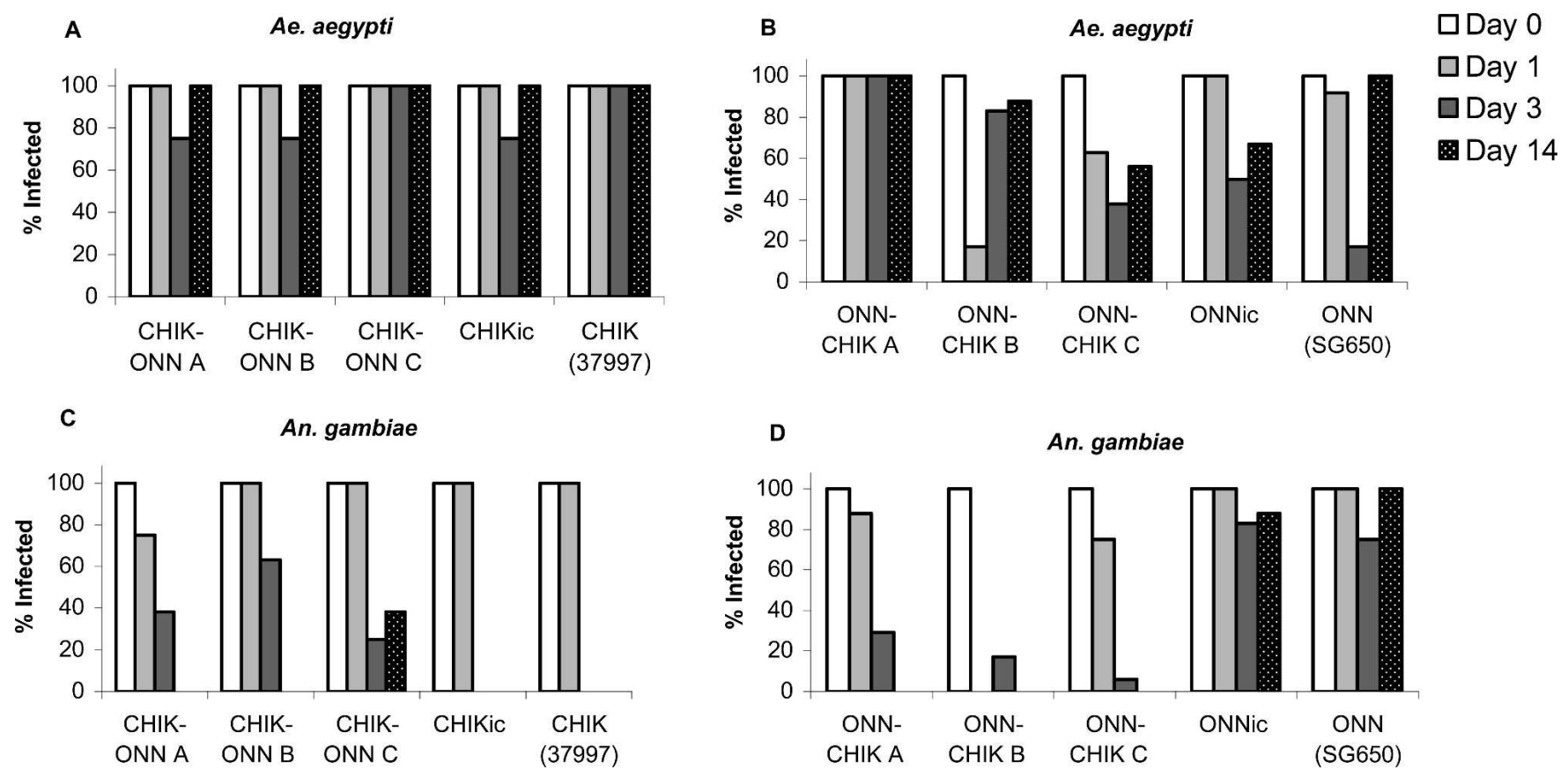

Figure 3. Percentage of orally infected Anopheles gambiae (A and $\mathbf{B})$ and Aedes aegypti $(\mathbf{C}$ and $\mathbf{D})$ mosquitoes analyzed by titration of whole mosquitoes on days $0,1,3$ and 14 post-infection (pi). Bloodmeal titers: CHIK-ONN A $6.52 \log _{10} \mathrm{TCID}_{50} / \mathrm{mL}$ CHIK-ONN B $6.52 \log _{10}$ TCID $50 / \mathrm{mL}$ CHIK-ONN C $7.52 \log _{10}$ TCID $_{50} / \mathrm{mL}$; ONN-CHIK A $7.52 \log _{10}$ TCID $_{50} / \mathrm{mL} ;$ ONN-CHIK B $6.95 \log _{10}$ TCID 50 /mL; ONN-CHIK C 6.95 $\log _{10} \mathrm{TCID}_{50} / \mathrm{mL}$. Mosquitoes analyzed per time point: three mosquitoes on day 0 pi; eight mosquitoes on days $1,2,3,7$, and 14 pi (except for ONN-CHIK B, which had six mosquitoes for days 2,3, and 7 pi and ONN-CHIK A, which had seven mosquitoes on day 2 pi for Ae. aegypti and day 3 pi for An. gambiae. Titers and number of mosquitoes analyzed for pCHIKic, CHIKV (37997), pONNic, and ONNV (SG650) are shown in Figure 2. For definitions of other abbreviations, see Figures 1 and 2.

infection rates in Ae. aegypti between ONN-CHIK B and ONN-CHIK A or ONN-CHIK B and ONN-CHIK C (Figure 3B).

The interaction of the capsid and the E2 cytoplasmic domain, which is important during virus assembly, was evaluated using two sets of chimeras. The first set contained chimeric viruses that derived the capsid and the E2 cytoplasmic domain from the same virus, either CHIKV or ONNV (CHIK-ONN A and ONN-CHIK A) (Figure 1). The second set contained chimeric viruses containing the capsid and E2 cytoplasmic domain from different viruses (CHIK-ONN B and ONN-CHIK B) (Figure 1). No significant difference $(P>$ $0.05)$ was found in day 14 pi infection rates in Ae. aegypti or An. gambiae infected with CHIKV nonstructural gene chimeras with both capsid and cytoplasmic domain genes from the same virus or from different viruses (Figure $3 \mathrm{~A}$ and $\mathrm{C}$ ). Infection rates in Ae. aegypti and An. gambiae with the reciprocal clones containing the nonstructural genes from ONNV also did not have a statistically different infection rates on day 14 pi (Figure 3B and D).
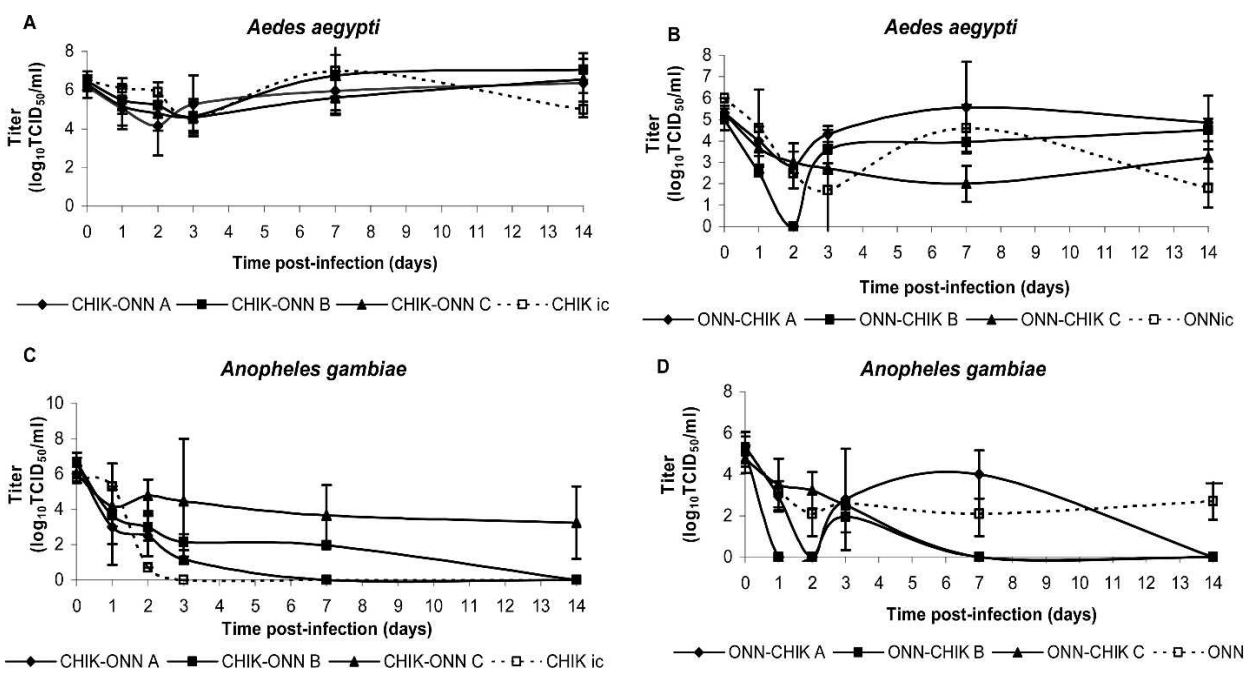

FIGURE 4. Viral titers and growth of viruses over time after a blood meal containing chimeric viruses and virus derived from the full-length pCHIKic or pONNic in Aedes aegypti $(\mathbf{A}$ and $\mathbf{B})$ and Anopheles gambiae $(\mathbf{C}$ and $\mathbf{D})$ mosquitoes. Blood meal titers and number of mosquitoes analyzed are shown in Figures 2 and 3. For definitions of abbreviations, see Figures 1 and 2. 
The chimeric virus containing the CHIKV E1 and E2 structural genes with the ONNV capsid and nonstructural genes (ONN-CHIK A) (Figure 1) had a significantly increased infection rate $(P<0.05)$ in $A$ e. aegypti mosquitoes on day $14 \mathrm{pi}$ compared with the chimera with the entire set of structural genes from CHIKV (ONN-CHIK C) (Figure 3B). The reciprocal chimeras containing the $\mathrm{CHIKV}$ nonstructural genes and either the capsid of CHIKV with the E1 and E2 of ONNV (CHIK-ONN A and B) or the complete ONNV structural genes (CHIK-ONN C) did not infect Ae. aegypti at a significantly different rate $(P>0.05)$ (Figure $3 \mathrm{~A})$.

The viral titers of the six chimeric viruses were compared in vivo in Ae. aegypti and An. gambiae mosquitoes (Figure 4). The chimeric viruses containing the CHIKV nonstructural genes (CHIK-ONN A, B, and C) produced growth curves that were similar to each other in Ae. aegypti (Figure 4A). The CHIK-ONN A and B chimeric viruses also showed similar growth characteristics in An. gambiae mosquitoes (Figure 4C). Virus derived from CHIK-ONN C was the only chimeric virus to replicate in $A n$. gambiae to day 14 pi (Figure 4C). The reciprocal chimeric viruses, which contained the nonstructural genes from ONNV, were all able to infect Ae. aegypti mosquitoes (Figure 4B), but were unable to infect An. gambiae mosquitoes on day 14 pi (Figure 4D).

Dissemination rates of viruses in Ae. aegypti and An. gambiae were analyzed by IFA of dissected salivary glands on days 7 and 14 pi (Table 1). Viruses derived from the fulllength infectious clones of CHIKV and ONNV and the six chimeric viruses were found to disseminate from $20 \%$ to $100 \%$ in Ae. aegypti mosquitoes on day 14 pi (Table 1). No significant differences were found between the three clones containing the CHIKV nonstructural genes with all or part of the ONNV structural genes (CHIK-ONN A, B, and C) in Ae. aegypti. Virus derived from these clones disseminated in 80 $100 \%$ of Ae. aegypti on day 14 pi. The substitution of all structural genes from CHIKV (ONN-CHIK C) dramatically decreased the dissemination rate in Ae. aegypti to $20 \%$ on day 14 pi. Significantly fewer $(P>0.05) A e$. aegypti mosquitoes

TABLE 1

Dissemination rates based on antigen detection in salivary glands by IFA from virus derived from full-length pCHIKic, pONNic, and six chimeric viruses in Aedes aegypti and Anopheles gambiae mosquitoes*

\begin{tabular}{cccc}
\hline $\begin{array}{c}\text { Virus } \\
\text { (blood meal titer) } \dagger\end{array}$ & Day pi & $\begin{array}{c}\text { Ae. aegypti } \\
\text { disseminated } \\
\text { Positive/total }(\%)\end{array}$ & $\begin{array}{c}\text { An. gambiae } \\
\text { disseminated } \\
\text { Positive/total }(\%)\end{array}$ \\
\hline CHIKic (7.95) & 7 & $9 / 10(90)$ & $0 / 10(0)$ \\
CHIK-ONN A & 14 & $5 / 10(50)$ & $0 / 10(0)$ \\
$(6.52)$ & 7 & $6 / 10(60)$ & $0 / 10(0)$ \\
CHIK-ONN B & 14 & $9 / 10(90)$ & $0 / 10(0)$ \\
$(6.52)$ & 7 & $4 / 10(40)$ & $0 / 10(0)$ \\
CHIK-ONN C & 14 & $10 / 10(100)$ & $0 / 10(0)$ \\
$(7.52)$ & 7 & $4 / 10(40)$ & $0 / 10(0)$ \\
ONN-CHIK C & 14 & $8 / 10(80)$ & $4 / 10(40)$ \\
$(6.95)$ & 7 & $2 / 10(20)$ & $0 / 10(0)$ \\
ONN-CHIK B & 14 & $2 / 10(20)$ & $0 / 10(0)$ \\
$(6.95)$ & 7 & $5 / 5(100)$ & $0 / 10(0)$ \\
ONN-CHIK A & 14 & $9 / 10(90)$ & $0 / 10(0)$ \\
$(7.52)$ & 7 & $8 / 10(80)$ & $0 / 10(0)$ \\
ONNic (6.52) & 7 & $8 / 10(80)$ & $0 / 10(0)$ \\
& 14 & $0 / 5(0)$ & $1 / 10(10)$ \\
\hline * pCHIKic = chikungunya infectious clone; pONNic $=$ o'nyong nyong infectious clone; & $3 / 5(60)$ & $10 / 10(100)$ \\
pi $=$ postinfection. & & &
\end{tabular}

were found to have a disseminated infection with virus derived from the ONN-CHIK C infectious clone compared with all other chimeras (Table 1). Only viruses containing the full structural genes of ONNV (virus derived from pONNic and CHIK-ONN C) were found to disseminate in An. gambiae on day 14 pi. Virus derived from pONNic disseminated in $100 \%$ of An. gambiae examined compared with CHIK-ONN C virus, which disseminated in $40 \%$ of the mosquitoes (Table 1 ).

\section{DISCUSSION}

The chimeric viruses containing ONNV and CHIKV genes that were produced for this project are valid tools to study the determinants of infection in Ae. aegypti and An. gambiae mosquitoes. The host specificities for the parental viruses and the viruses derived from full-length infectious clones were similar. Therefore, differences in host specificity of the chimeric viruses are due to the nucleotide substitutions within the structural genes. The full-length clones and the six chimeras were infectious to Ae. aegypti mosquitoes on day $14 \mathrm{pi}$, which indicates that all the viruses are viable and able to replicate to sufficient titers to produce infection and to disseminate in this mosquito species. Day 14 pi viral replication and dissemination data are used to standardize comparisons and to ensure that virus is replicating in the mosquitoes analyzed. Earlier time points may only indicate exposure to the virus and not viral replication.

The virus titers obtained from the full-length pONNic and ONNV (SG650) were not statistically different in An. gambiae mosquitoes on day 14 pi (Figure 2D). Therefore, this infectious clone is suitable for analysis of ONNV genes that could potentially expand the host range of CHIKV to infect An. gambiae mosquitoes. The day 14 pi viral titer from pONNic was not as high in Ae. aegypti mosquitoes as the ONNV (SG650) (Fgiure 2B); however, the infectious clone did infect most $(67 \%)$ of mosquitoes examined at this time point (Figure 3B). Infection and dissemination rates for the virus derived from full-length pCHIKic were not statistically different from the parental virus at day 14 pi in both of the mosquito species examined, thereby enabling accurate comparisons of CHIK-ONN chimeras using this clone (Figure 2A and C). Three day 7 pi An. gambiae mosquitoes were infected with CHIKV, whereas none of the pCHIKic mosquitoes were infected past day 3 pi. This may indicate limited, low titer, replication of the parental virus in An. gambiae; however, neither virus was able to infect An. gambiae on day 14 pi (Figure 2C).

Chimeric viruses are not necessarily less infectious than virus derived from a full-length infectious clone. Comparison of the chimeric viruses with virus derived from full-length pONNic or pCHIKic showed that there was no significant effect on the ability of the chimeric viruses to infect $A e$. aegypti mosquitoes on day 14 pi (Figure $3 \mathrm{~A}$ and $\mathrm{B}$ ). The only chimeric virus that had a decreased infection rate compared with the viruses derived from either $\mathrm{pONNic}$ or $\mathrm{pCHIKic}$ was the ONN-CHIK C chimera (Figure 3B). This chimera infected $56 \%$ of Ae. aegypti on day 14 pi compared with the virus derived from pONNic and pCHIKic, which infected $67 \%$ and $100 \%$ of mosquitoes analyzed (Figure $3 \mathrm{~A}$ and $\mathrm{B}$ ). These data are in contrast to previous studies of chimeras based on Sindbis virus (SINV) and Ross River virus (RRV), which indicated that there is a reduced ability of chimeric 
alphaviruses to replicate in various cell lines. ${ }^{26}$ The chimeric SIN-RR viruses did not replicate as well in cell culture as the parental viruses. It was concluded that the chimeras were defective in their interactions between the nonstructural and structural regions leading to inefficient RNA replication. ${ }^{27}$ Our study suggests this to be a virus-specific phenomenon.

Four additional chimeras were produced to examine the role of the individual structural genes in determining host specificity. Chimeric viruses to evaluate the interaction between the E2 cytoplasmic domain and the capsid protein were examined in Ae. aegypti and An. gambiae (Figure 1). This interaction is important in the late stages of virus assembly within the cell. ${ }^{27} \mathrm{~A}$ chimeric RRV in which the capsid sequence was replaced with the capsid of SINV had reduced virion production. ${ }^{28}$ Virion production increased when seven amino acids located in the cytoplasmic domain of the E2 gene were replaced with SINV sequence. ${ }^{28}$ Virus that was defective in the capsid and E2 glycoprotein interactions was deficient in budding. ${ }^{28}$ In contrast, chimeras of SINV containing capsid sequence from either RRV or Simliki Forest virus did not have reduced virion production. ${ }^{29}$ In our study, chimeric viruses of ONNV and CHIKV containing the capsid and the cytoplasmic domain of either the same virus or of a different virus (Figure 1) did not show a significant change in the infection rate of Ae. aegypti or An. gambiae on day 14 pi (Figure 3 ). The dissemination rates of these chimeras were similar, with CHIK-ONN A and B disseminating in $90 \%$ and $100 \%$ of the Ae. aegypti, respectively, and in $0 \%$ of the An. gambiae examined on day 14 pi (Table 1). The reciprocal chimeric viruses ONN-CHIK A and B had similar infection and dissemination rates in both the Ae. aegypti and An. gambiae mosquitoes analyzed (Figure $3 \mathrm{~B}$ and $\mathrm{D}$ and Table 1). ONNCHIK A and B disseminated in $80 \%$ and $90 \%$ of the $A e$. aegypti analyzed and in $0 \%$ of the An. gambiae analyzed (Table 1). These results indicate that the capsid and cytoplasmic domain interactions in ONNV and CHIKV do not play a role in the ability of these viruses to infect different species of mosquitoes.

The primary molecular determinant of viral host range enabling infection of An. gambiae is found in all of the structural genes of ONNV. Virus derived from the full-length pCHIKic and virus from three chimeras containing the CHIKV nonstructural genes (CHIK-ONN A, B, and C) infected $100 \%$ of Ae. aegypti on day $14 \mathrm{pi}$ (Figure 3A). A significant difference $(P<0.05)$ was found between the virus derived from pCHIKic and CHIK-ONN C on day 14 pi in An. gambiae (Figure $3 \mathrm{C}$ ). None of the mosquitoes analyzed at this time point were infected with the full length pCHIKic virus whereas, $38 \%$ of An. gambiae were infected with virus derived from CHIK-ONN C (Figure 3C). The dissemination rates were high for virus derived from CHIK-ONN A, B, C and pCHIKic in Ae. aegypti (Table 1). Virus derived from CHIK-ONN C was the only chimeric virus to disseminate in An. gambiae (Table 1). The virus derived from full-length pONNic was highly infectious and had a high dissemination rate in An. gambiae on day 14 pi (Figure 4D and Table 1). These data demonstrate that the ONNV structural genes are important in allowing infection and dissemination in An. gambiae mosquitoes and indicate that substitution of partial structural genes from ONNV do not change the host range of CHIKV.

The chimeric infectious clones produced for this research represent useful tools for the investigation of the molecular basis of virus-vector relationships, and will facilitate further studies to identify the role of specific nucleotides in the infection and dissemination of arboviruses in mosquitoes. Additional chimeric viruses, such as ones examining the $5^{\prime}$ and $3^{\prime}$ NCRs and individual nonstructural genes, will enable further studies of viral host determinants of these viruses. The only chimeric virus produced from this study that was able to infect An. gambiae mosquitoes was the chimeric virus containing viral genes encoding all of the structural proteins of ONNV. The ONNV nonstructural genes enhance viral infection of An. gambiae mosquitoes, as indicated by the reduced infection rate of the chimeric virus containing the CHIKV nonstructural genes with the ONNV structural genes. However, the ONNV viral structural proteins alone enable the chimeric CHIK-ONN C virus to infect An. gambiae mosquitoes.

Received September 20, 2005. Accepted for publication November 19, 2005.

Acknowledgments: We thank Jing Haung for her expert technical assistance with this project, Dr. Robert Tesh for providing the CHIKV used in this study, and Dr. Ann Powers for some of the ideas used in this study.

Financial support: This work was supported in part by National Institutes of Health grant AI47877 originally awarded to Dr. Ann Powers. Kate L. McElroy was supported by a Centers for Disease Control and Prevention Fellowship Training Program in Vector-Borne Infectious Diseases TOI/CCT622892.

Authors' addresses: Dana L. Vanlandingham, Konstantin Tsetsarkin, Kimberly A. Klinger, Chao Hong, Kate L. McElroy, and Stephen Higgs, Department of Pathology, University of Texas Medical Branch, Keiller 2.104 L 20762, 301 University Boulevard, Galveston, TX 77555-0609, Telephone: 409-747-2426, Fax: 409-772-2511, E-mail: dlvanlan@utmb.edu. Michael J. Lehane, Liverpool School of Tropical Medicine, Pembroke Place, Liverpool L3 5QA, United Kingdom, Telephone: 44-151-705- 3316 (Office), 44-151-705-3231 (Laboratory), Fax: 44-151-705-3369.

\section{REFERENCES}

1. Karabatsos N, 1985. International Catalogue of Arboviruses. Third edition. San Antonio, TX: American Society of Tropical Medicine and Hygiene.

2. Strauss JH, Strauss EG, 1994. The alphaviruses: gene expression, replication, and evolution. Microbiol Rev 58: 491-562.

3. Johnson BK, 1988. O'nyong-nyong virus disease. Monath TP, ed. The Arboviruses: Epidemiology and Ecology. Volume II. Boca Raton, FL: CRC Press, 217-223.

4. Williams MC, Woodall JP, Corbet PS, Gillett JD, 1965. O'nyongnyong fever: an epidemic virus disease in East Africa. VIII. Virus isolations from Anopheles mosquitoes. Trans $R$ Soc Trop Med Hyg 59: 300-306.

5. Williams MC, Woodall JP, 1961. O'nyong nyong fever: an epidemic virus disease in east Africa: isolation and some properties of the virus. Trans R Soc Trop Med Hyg 55: 135-141.

6. Lanciotti RS, Ludwig ML, Rwaguma EB, Lutwama JJ, Kram T, Karabatsos N, Cropp BC, Miller BR, 1998. Emergence of O'nyong-nyong fever in Uganda after a 35-year absence: genetic characterization of the virus. Virol 252: 258-268.

7. Powers AM, Brault AC, Tesh RB, Weaver SC, 2000. Reemergence of chikungunya and o'nyong-nyong viruses: evidence for distinct geographical lineages and distant evolutionary relationships. J Gen Virol 81: 471-479.

8. Haddow AJ, Davies CW, Walker AJ, 1960. O'nyong nyong fever: an epidemic virus disease in east Africa: introduction. Trans $R$ Soc Trop Med Hyg 54: 517-522.

9. Rwaguma E, Lutwama JJ, Sempala SDK, Kiwanuka N, Kamugisha J, Okware S, Bagambisa G, Lanciotti R, Roehrig JT, Gubler DJ, 1997. Emergence of epidemic O'nyong nyong fever 
in southwestern Uganda after an absence of 35 years (letter). Emerg Infect Dis 3: 77.

10. Tesh RB, 1982. Arthritides caused by mosquito-borne viruses. Annu Rev Med 33: 31-40.

11. Pavri KM, 1964. Presence of chikungunya antibodies in human sera collected from Calcutta and Jamshedpur before 1963. Indian J Med Res 52: 698-702.

12. Jupp PG, McIntosh BM, 1988. Chikungunya virus disease. Monath TP, ed. The Arboviruses: Epidemiology and Ecology. Volume II. Boca Raton, FL: CRC Press, 137-157.

13. Laras K, Sukri NC, Larasati RP, Bangs MJ, Kosim R, Djauzi, Wandra T, Master J, Kosasih H, Hartati S, Beckett C, Sedyaningsih ER, Beecham HJ III, Corwin AL, 2005. Tracking the re-emergence of epidemic chikungunya virus in Indonesia. Trans $R$ Soc Trop Med Hyg. 99: 128-141.

14. Halstead SB, Scanlon JE, Umpaivit P, Udomsakdi S, 1969. Dengue and Chikungunya virus infection in man in Thailand, 19621964. IV. Epidemiologic studies in the Bankok metropolitan area. Am J Trop Med Hyg 18: 997-1021.

15. Rao TR, 1966. Recent epidemics caused by Chikungunya virus in India, 1963-1965. Sci Culture 32: 215.

16. Porter KR, Tan R, Istary Y, Suharyono W, Sutaryo, Widjaja S, Ma'Roef C, Listiyaningsih E, Kosasih H, Hueston L, McArdle J, Juffrie M, 2004. A serological study of Chikungunya virus transmission in Yogyakarta, Indonesia: evidence for the first outbreak since 1982. Southeast Asian J Trop Med Public Health 35: 408-415.

17. Pastorino B, Muyembe-Tamfum JJ, Bessaud M, Tock F, Tolou H, Durand JP, Peyrefitte CN, 2004. Epidemic resurgence of Chikungunya virus in Democratic Republic of the Congo: identification of a new central African strain. J Med Virol 74: 277-282.

18. Vanlandingham DL, Hong C, Klingler K, Tsetsarkin K, McElroy KL, Powers AM, Lehane MJ, Higgs S, 2005. Differential infectivities of o'nyong nyong and chikungunya virus isolates in Anopheles gambiae and Aedes aegypti mosquitoes. Am J Trop Med Hyg. 72: 616-621.

19. Vanlandingham DL, Tsetsarkin K, Hong C, Klingler K, McElroy KL, Lehane MJ, Higgs S, 2005. Development and characterization of a double subgenomic chikungunya virus infectious clone to express heterologous genes in Aedes aegypti mosquitoes. Insect Biochem Mol Biol 35: 1162-1170.

20. Brault AC, Foy BD, Myles KM, Kelly CL, Higgs S, Weaver SC, Olson KE, Miller BR, Powers AM, 2004. Infection patterns of o'nyong nyong virus in the malaria-transmitting mosquito, Anopheles gambiae. Insect Mol Biol 13: 625-635.

21. Higgs S, Olson KE, Kamrud KI, Powers AM, Beaty BJ, 1997. Viral expression systems and viral infections in insects. Crampton JM, Beard CB, Louis C, eds. The Molecular Biology of Disease Vectors: A Methods Manual. London: Chapman and Hall, 457-483.

22. Woodward TM, Miller BR, Beaty BJ, Trent DW, Roehrig JT, 1991. A single amino acid change in the E2 glycoprotein of Venezuelan equine encephalitis virus affects replication and dissemination in Aedes aegypti mosquitoes. J Gen Virol 72: 2431-2435.

23. Miller BR, Mitchell CJ, 1991. Genetic selection of a flavivirusrefractory strain of the yellow fever mosquito Aedes aegypti. Am J Trop Med Hyg 45: 399-407.

24. Higgs S, Beaty BJ, 1996. Rearing and containment of arthropod vectors. Marquardt WC, Beaty BJ, eds. Biology of Disease Vectors. Niwot, CO: University Press of Colorado, 595605.

25. Cosgrove JB, Wood RJ, Petric D, Evans DT, Abbott RH, 1994 A convenient mosquito membrane feeding system. J Am Mosq Control Assoc 10: 434-436.

26. Kuhn RJ, Griffin DE, Owen KE, Niesters HG, Strauss JH, 1996. Chimeric Sindbis-Ross River viruses to study interactions between alphavirus nonstructural and structural regions. $J$ Virol 70: 7900-7909.

27. Wilkinson TA, Tellinghuisen TL, Kuhn RJ, Post CB, 2005. Association of Sindbis virus capsid protein with phospholipid membranes and the E2 glycoprotein: implications for alphavirus assembly. Biochemistry 44: 2800-2810.

28. Lopez S, Yao JS, Kuhn RJ, Strauss EG, Strauss JH, 1994. Nucleocapsid-glycoprotein interactions required for assembly of alphaviruses. J Virol 68: 1316-1323.

29. Frolov I, Frolova E, Schlesinger S, 1997. Sindbis virus replicons and Sindbis virus: assembly of chimeras and of particles deficient in virus RNA. J Virol 71: 2819-2829. 